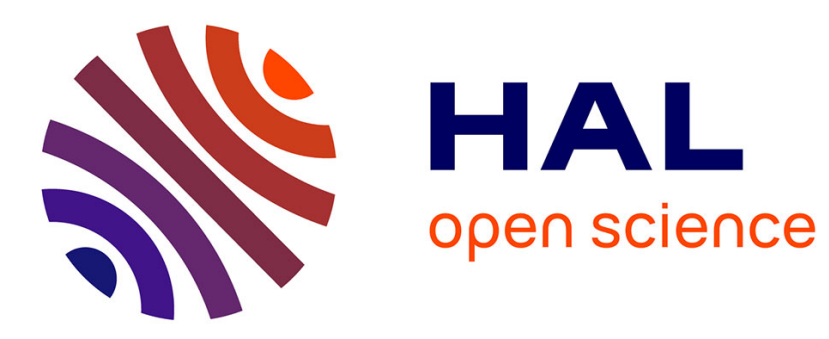

\title{
Matching CAD model and image features for robot navigation and inspection of an aircraft
}

Igor Jovančević, Ilisio Viana, Jean-José Orteu, Thierry Sentenac, Stanislas Larnier

\section{- To cite this version:}

Igor Jovančević, Ilisio Viana, Jean-José Orteu, Thierry Sentenac, Stanislas Larnier. Matching CAD model and image features for robot navigation and inspection of an aircraft. 5th International Conference on Pattern Recognition Applications and Methods (ICPRAM'2016), Feb 2016, Roma, Italy. 10.5220/0005756303590366 . hal-01353317

\section{HAL Id: hal-01353317 https://hal.science/hal-01353317}

Submitted on 11 Aug 2016

HAL is a multi-disciplinary open access archive for the deposit and dissemination of scientific research documents, whether they are published or not. The documents may come from teaching and research institutions in France or abroad, or from public or private research centers.
L'archive ouverte pluridisciplinaire HAL, est destinée au dépôt et à la diffusion de documents scientifiques de niveau recherche, publiés ou non, émanant des établissements d'enseignement et de recherche français ou étrangers, des laboratoires publics ou privés. 


\title{
Matching CAD model and image features for robot navigation and inspection of an aircraft
}

\author{
Igor Jovančević ${ }^{1}$, Ilisio Viana ${ }^{1}$, Jean-José Orteu ${ }^{1}$, Thierry Sentenac ${ }^{1,2,3}$, Stanislas Larnier ${ }^{2,3}$ \\ ${ }^{1}$ Université de Toulouse, CNRS, INSA, UPS, Mines Albi, ISAE, ICA, Campus Jarlard, F-81013 Albi, France \\ ${ }^{2}$ CNRS, LAAS, 7 Avenue du Colonel Roche, F-31400 Toulouse, France \\ ${ }^{2}$ Université de Toulouse, UPS, LAAS, F-31400 Toulouse, France \\ \{igor.jovancevic, ilisio.viana, jean-jose.orteu,thiery.sentenac\}@mines-albi.fr, slarnier@laas.fr
}

\begin{abstract}
Keywords: computer-aided-inspection, image analysis, feature matching, PTZ camera, Hough transform, EDCircle
Abstract: $\quad$ This paper focuses on the navigation of a moving robot equipped with cameras, moving around an aircraft to perform inspection of different types of items (probes, doors, etc.). Matching CAD model and image features is useful to provide meaningful features for localization and inspection tasks. In our approach two primitive sets are matched using a similarity function. The similarity scores are injected in the edges of a bipartite graph. A best-match search procedure in bipartite graph guarantees the uniqueness of the match solution. The method provides good matching results even when the location of the robot with respect to the aircraft is badly estimated. Inspection approaches on static ports and air inlet vent are presented.
\end{abstract}

\section{INTRODUCTION}

Airplanes are periodically inspected, either on the airport tarmac between flights or in a hangar during maintenance operations. Today, these inspections are done by human operators, mainly visually and sometimes with some inspection tools. In order to make the inspection quicker, more exhaustive and more accurate, as well as for traceability reasons, a multipartners research project is being carried on to develop a mobile robot equipped with several sensors (cameras and 3D scanners) to perform an automatic inspection of an airplane. The chosen approach is based on an autonomous mobile robot on the ground, capable to communicate with human operators and infrastructures around the aircraft. In addition to the long range navigation task, at each checkpoint, the robot performs an inspection task of several items (probes, doors, etc.).

Robotic airplane inspection from the ground, based on computer vision, is a specific application not enough studied. Previous efforts (Siegel et al., 1998; Mumtaz et al., 2012) were mainly focused on detailed airplane skin inspection with robot crawling on the airplane surface. In most cases, industrial automation systems (Malamas et al., 2003) are used to inspect known objects at fixed positions with appropriate illumination necessary to extract meaningful features. In our application, sensors are mounted on the moving robot and no additional illumination is used. Moreover, our intention is to enable robot to cover as many items as possible from one position, i.e. to move as less as possible.

Firstly, navigation algorithms lead the robot to a checking position which is localized in the aircraft model. Secondly, automated image acquisition is provided by controlling Pan-Tilt-Zoom (PTZ) camera and pointing it towards the item to be inspected. Inspection is performed by processing the images. For each item, integrity or right configuration should be verified. To perform more accurately, it is required to obtain a zoomed view of the element to be inspected. Therefore, the essential step is detection of the region of interest (ROI) in an image. Detection of items on the aircraft surface is an important step for navigation tasks as well. Advantage in the present context is that the airplane 3D model with the items to be detected is known (Fig. 1). Therefore, it is possible to project it onto the image plane to obtain the expected shape and position of desired items.

Since the shapes on the airplane surface are regular geometrical shapes such as circles, ellipses or rounded corners rectangles, our detection approaches are relying on geometric feature extraction techniques. Extracted primitives are ellipses and straight line segments. After extraction, each primitive is associated with an item or part of an item. Our approach 


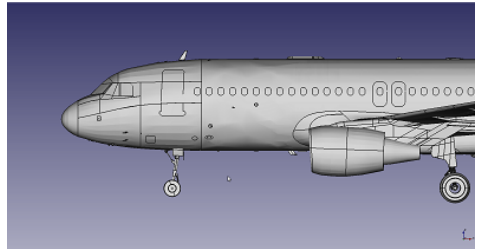

(a) Aircraft model with a far away view.

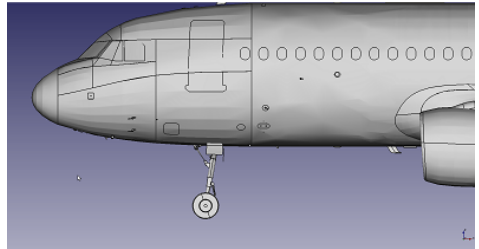

(b) Aircraft model near the checking position.

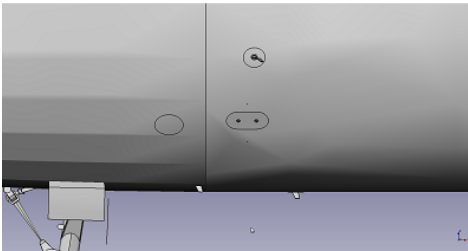

(c) Aircraft model view from the checking position.

Figure 1: Screenshots of the 3D aircraft model.

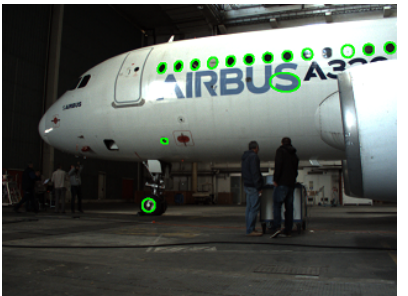

(a) Navigation far from the aircraft.

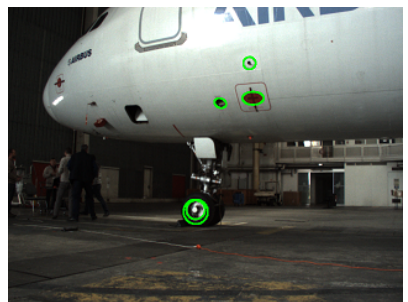

(b) Navigation near the aircraft.

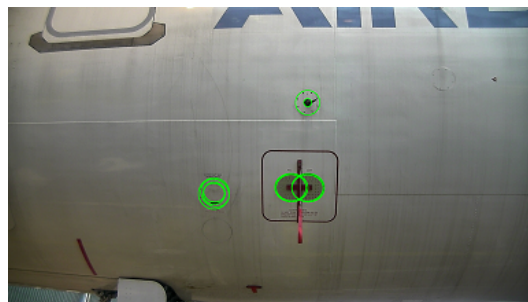

(c) Inpection of the static port element.

Figure 2: Aircraft views taken by the mobile robot for navigation and inspection. Ellipse feature extraction with EDCircle.

proposes a score for similarity between the projections and the primitives detected in the real image.

Navigation algorithms around the aircraft rely firstly on visual servoing (Chaumette and Hutchinson, 2006). To perform visual servoing between checkpoints around an aircraft, the navigation algorithm needs features associated with aircraft visual elements (windows, tires, trapdoors, company logo, etc.). Since the aircraft inspection has to be performed inside or outside, at any time of the day and with different weather conditions, the detection part has to work even with low contrast images.

Different tracking methods are currently used such as low contrast, blob, motion edges, template. The low contrast tracker is a succession of low contrast detections. The search zone takes into account the previous location of the target and the robot movements. The other methods come from the ViSP library (Marchand and Chaumette, 2005). Our strategy is to let the robot choose the best tracking method in function of the aircraft visual element and its status in the scene. The selection of the best tracking method is done by taking into account knowledge acquired from experiments and detection results.

During navigation, robot can switch from visual servoing to obstacle avoidance (Futterlieb et al., 2014). In this mode, the plane could dissapear from the field of view and the robot location is updated only with odometry. Then a new detection and matching of the features is needed to restart the visual servoing and correct the robot location. The visual servoing algorithm leads the robot to a checking position.

Final step in the process is visual inspection and making decision on the state of a particular item on the aircraft. Usual weakness of an inspection system is being specialized in inspecting one particular type of object. In that sense, each of the items on the airplane could be an isolated, specific, inspection problem. Our intention is to design more general strategies, applicable to a class of similar items by changing just parameters and geometry. For instance, there are many doors and vents on the airplane which can be closed or open. For more details about inspection, reader is referred to (Jovančević et al., 2015).

Navigation cameras acquire color images at a resolution of $480 \times 640$ pixels. PTZ camera provides color images at a resolution of $1080 \times 1920$ pixels. Acquisition images of the aircraft and associated views of the 3D model are presented in Fig. 2 and 1 respectively. The three images present the distance ranging from far away to a checking position of the static port element. Fig. 2a is an image coming from the navigation camera, taken when the mobile robot is far away from the airplane. Fig. $2 \mathrm{~b}$ is also an image coming from the navigation camera, taken when the mobile robot is near the airplane. Fig. $2 \mathrm{c}$ is an image coming from the PTZ camera, taken when the mobile robot stopped at the end of the visual servoing and it should perform inspection.

Paper is organized as follows. Two used feature ex- 
traction algorithms are introduced in Sec. 2. In Sec. 3 we explain the matching method between features extracted from the computer-aided design (CAD) model and the ones detected in the image. The matching results for the image views from Fig. 2 are given in Sec. 4. Two inspection methods relying on clues such as uniformity of isolated image regions and convexity of segmented shapes are presented in Sec. 5.

\section{FEATURE EXTRACTION}

\subsection{Ellipses Extraction}

Akinlar and Topal have proposed a robust circle detection method (Akinlar and Topal, 2013), named EDCircles. The ellipses features in camera images are obtained after postprocessing of EDCircles results (Fig. 2). Since it is impossible to have an intersection of two circles on the airplane, intersected circles are merged and an ellipse is fitted on them (Prasad et al., 2013). Sometimes, there is multiple results on the same location and they are also merged. See the result of postprocessing in Fig. 4c, 5c and 7c.

Ellipse detector is employed in order to detect numerous elliptical shapes on the airplane skin such as Air inlet vent (Sec. 5.1).

\subsection{Line Segments Extraction}

For segments extraction, we are running the progressive probabilistic Hough Transform (PPHT) (Matas et al., 2000) implementation from OpenCV library. This method provides set of continuous straight line segments. It is possible to use other segment detectors such as more recent LSD (von Gioi et al., 2010) or EDLines (Akinlar and Topal, 2011).

Outputs of all these detectors demand sensitive postprocessing step of filtering due to the large set of detected segments (Fig. 3a). First, segments which are aligned (approximately belong to one ray) are identified and average ray is calculated. Then, this set of aligned segments is projected onto the average ray and the projections are divided into clusters of overlapping (or nearby) segments. The segments of each such cluster are finally merged into one segment (Fig. 3b).

The resulting set of segments is traversed in order to detect some items on the airplane skin. For example, there is an interest to identify four segments which make rectangular shape such as static port (Sec. 5.2).

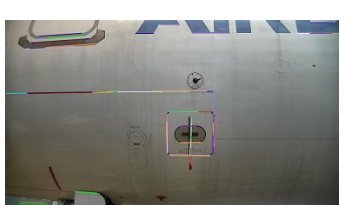

(a) Set of segments extracted by the PPHT method.

Figure 3: Line segments extraction.

\section{MATCHING OF PRIMITIVES}

\subsection{Feature Matching Using A Similarity Function}

Line segments matching has been a widely studied topic since segments are used in numerous applications such as 3D reconstruction (Bay et al., 2005). This is also the case for primitives like ellipses (Hutter and Brewer, 2009).

\subsubsection{Preliminaries}

Given two sets of primitives $X$ and $Y$, with $X$ representing primitives projected from the model and $Y$ representing primitives in the real image, we will show how a match score $s\left(X_{k}, Y_{m}\right)$ denoting the similarity between $X_{k} \in X$ and $Y_{m} \in Y$ can be computed.

\subsubsection{Basic Graph Theory Concepts}

Given a graph $G=(V, E)$, an edge $e \in E$ which links two nodes $u \in V$ and $v \in V$ is said to be incident to the nodes $u$ and $v$. A graph $G=(V, E)$ is said to be bipartite if $V(G)$ can be partitioned into two disjoint sets $X$ and $Y$ such that every edge $e \in E$ joins a vertex in $X$ to a vertex in $Y$.

\subsubsection{Similarity Function}

Two types of primitives are considered: ellipses and line segments. A line segment is associated with an attribute vector composed of the segment mid-point, the length and the orientation. An ellipse is described by its center, orientation and area. We divide the attribute vector in two classes $\left(c=\left[c_{1}, c_{2}\right]\right)$. Attributes such as area or length belong to the first group as we can compute their ratio (Eq. 1). Computing the ratio for attributes such as center coordinates or orientation is, however, not meaningful. Therefore, these attributes will be in class $c_{2}$. For such attributes, the similarity score is computed as a distance of the absolute difference from the maximal accepted disparity 
between the theoretical and the test primitive (Eq. 2). Maximal accepted disparity is preset by taking into account the distance between primitives in the model. The match function was proposed by (McIntosh and Mutch, 1988) for line matching. In case of line matching, the metrics used are the ratio of segment lengths, the absolute difference of the orientation and the euclidean distance, conditioned in such a manner that the score between two primitives is always $\leq 1$. We extended the concept in order to use it with ellipses as well. In this case we use the area of the ellipses instead of the length of the segment.

$$
\begin{gathered}
s_{i}= \begin{cases}\frac{\min \left(X_{k_{i}}, Y_{m_{i}}\right)}{\max \left(X_{k_{i}}, Y_{m_{i}}\right)}, & \text { if } i \in c_{1} \\
\frac{\delta_{i}-a b s\left(X_{k_{i}}-Y_{m_{i}}\right)}{\delta_{i}}, & \text { if } i \in c_{2}\end{cases} \\
s\left(X_{k}, Y_{m}\right)=\sum w_{i} s_{i}
\end{gathered}
$$

where $X_{k}$ stands for a primitive projected from the CAD model, $Y_{m}$ is a primitive in the real image and $i$ denotes the $i^{t h}$ attribute for a given primitive. $\delta_{i}$ is the maximal accepted disparity for the $i^{t h}$ attribute in the class $c_{2}$. The scalar $s_{i}$ is the match score between one theoretical primitive and one test primitive for the $i^{t h}$ attribute. Finally, $s\left(X_{k}, Y_{m}\right)$ denotes the match score between $X_{k}$ with $Y_{m}$. As some attributes may be more relevant than others (Jr. et al., 2005), a priority weighting function may be suitable $\left(w_{i}\right)$.

The matching is accomplished by exploring the entire sets of theoretical and real primitives. A matching matrix containing all the scores (Eq. 3) between primitives in the set $X$ with primitives in $Y$ is computed (Table 1) then used to construct the bipartite graph.

Table 1: Matching matrix for the case in Fig. 4.

\begin{tabular}{|c|c|c|c|c|c|}
\cline { 3 - 6 } \multicolumn{2}{c|}{} & \multicolumn{4}{c|}{ Test } \\
\cline { 2 - 6 } \multicolumn{2}{c|}{} & 1 & 2 & 3 & 4 \\
\hline \multirow{3}{*}{ Model } & 1 & -0.407 & $\mathbf{0 . 2 0 4}$ & -0.424 & -0.18 \\
\cline { 2 - 6 } & 2 & -1.147 & 0.446 & $\mathbf{0 . 4 6 1}$ & 0.193 \\
\cline { 2 - 6 } & 3 & -0.565 & 0.120 & -0.113 & $\mathbf{0 . 5 4 8}$ \\
\hline
\end{tabular}

The similarity function is conditioned to be in the range $[0,1]$ when $i \in c_{1}$. When $i \in c_{2}$ the score may be $<0$. In our application only candidates having a match score in the range $[0,1]$ are considered.

\subsection{Search Of Mutual Best Match In A Bipartite Graph}

Bipartite graph matching has been widely studied and has applications in various fields of science (Panda and Pradhan, 2013), (Fishkel et al., 2006). It is particularly suitable for a two-class matching problem. For more details on how this concept is used in our work, reader is referred to (Viana et al., 2015).

First we keep $M$ occurrences of a score in the matching matrix meeting a predefined threshold. A bipartite graph containing $M$ edges is then created. This graph holds two types of nodes $(X, Y)$, representing theoretical and real primitives respectively. A $X$-type node in the bipartite graph is linked with a $Y$-type node when their similarity score satisfies the threshold condition. The edge linking these two nodes is weighted with their similarity score (Eq. 3). Depending on the threshold value, one $X$-type node may be connected to more than one $Y$-type node and reversely.

Further, best matches are found for all the $X$-nodes. Let $X_{k}$ be a $X$-node. We search the edge incident to $X_{k}$, holding the maximal weight. At the end of this stage, if in the best match list there is more than one $X$-node matched to the same $Y$-node, we proceed to the second stage. Otherwise, we reached one-to-one correspondences for all CAD primitives, so the matching is done. In the second stage, we are searching for best match for each $Y$-node which was previously matched with more than one $X$-node. In these cases, we are keeping only the mutual best match. Other edges incident to $Y$-node are eliminated. Therefore, some of the $X$-nodes might stay unmatched. Fig. 6 shows all the possible edges between nodes of two classes (grey) and the best match for each of the $X$-nodes (blue). We have a conflict because $Y$-node 2 is chosen as a best match for two $X$-nodes 12 and 13. After computing the best match for the $Y$-node 2, we are keeping the edge $2-13$ and eliminating the edge $2-12$. Final matching result is presented in $5 \mathrm{~b}$.

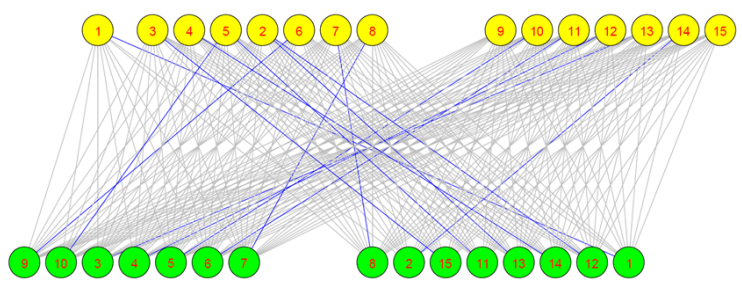

Figure 6: Conflict matches.

The mutual best match search guarantees the uniqueness constraint of the matching, i.e. each CAD primitive is matched to a different real primitive, or it is not matched at all.

\section{MATCHING RESULTS}

In this section the matching results are presented. Fig. 5, 4 and 7 concern ellipse matching. Fig. 8 concerns segments matching. For all, the model features 

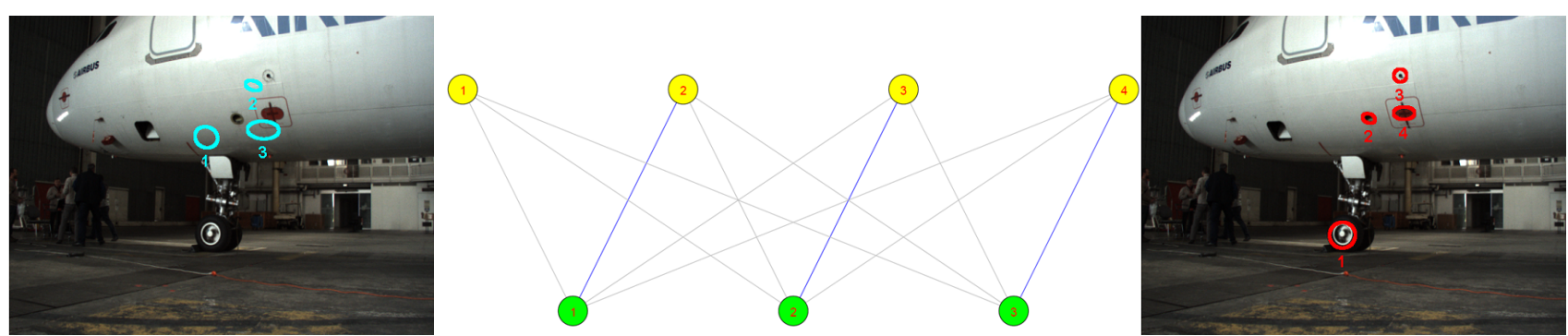

(a) Model features projected to the(b) Bipartite graph with eliminated edges (grey) and matches(c) Ellipse detection in the sensed imsensed image. (blue). age (EDCircles and our postprocessing step).

Figure 4: Navigation near the aircraft.
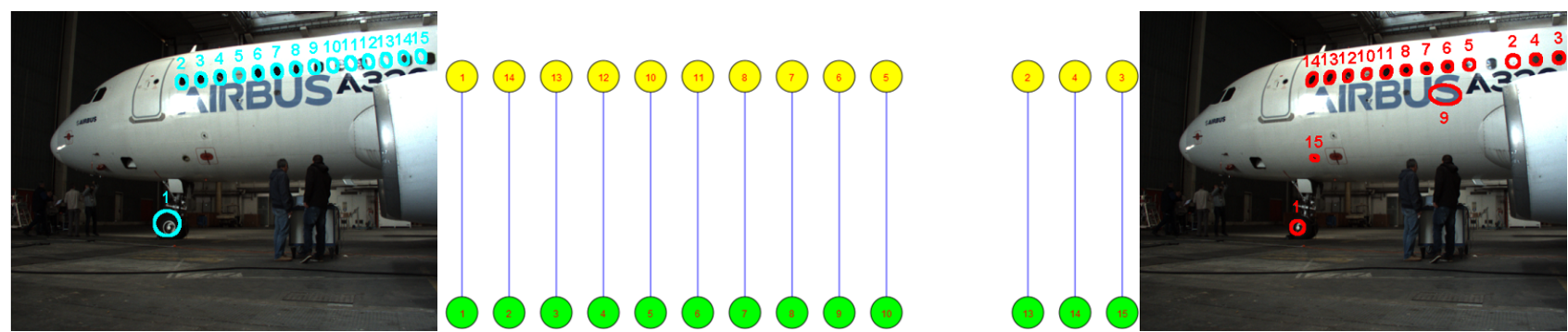

(a) Model features projected to the(b) Bipartite graph with matches (blue) (eliminated edges not(c) Ellipse detection in the sensed imsensed image. shown). age (EDCircles and our postprocessing step).

Figure 5: Navigation far from the aircraft.

are in cyan and the image ones are in red. In the graph, model features are presented as green circles and image ones as yellow circles. From a starting position, the robot has to detect the airplane then go to a checking area where it can inspect the air inlet vent and the static port (ellipse features 1 and 2 in Fig. 7c).

In Fig. 5, the robot is at its starting position. The aircraft location is roughly known from this position. The CAD model projection is presented in Fig. 5a. Only windows and front tire are projected. At this distance, the other ellipse features of the airplane are usually not detected. The projection is inaccurate for the most right windows. The ellipse image features are given in Fig. 5c. Some windows are not detected due to light conditions. Undesired ellipses are the air inlet vent (feature 15) and a part of the logo (feature 9). The bipartite graph in Fig. 5b resumes the matching. The left part is made of 10 good matches and the right part of 3 bad matches. Last three are inaccurate matches because windows are actually projected on their precedent neighbors. These three can be ignored by taking into account the distance between the windows. The robot starts the visual servoing.

In Fig. 4, the robot performed a spiral obstacle avoidance, to avoid moving humans. The tracking algo- rithms lost targets. Relying on odometry, the robot has to detect some features again, taking into account its estimated location. The CAD model projection is presented in Fig. 4a. The air inlet vent, the probe area and the static port are projected (respectively features 1, 2 and 3). The projection is slightly inaccurate. The ellipse image features are given in Fig. 4c. An undesired ellipse is extracted from the tire (feature 1). Fig. 4b, the bipartite graph resumes the perfect ellipse matching. The robot restarts the visual servoing.

In Fig. 7 and 8, the robot arrives in a checking area. The CAD model projection is presented in Fig. 7a and 8a. For the ellipse features, the air inlet vent, the probe area and the static port are projected (respectively features 1, 2 and 3). For the segments, the red warning zone around the static port is projected. Unfortunately due to bad robot pose estimation, the projection is inaccurate. The ellipse image features are given in Fig. 7c. The segment image features are given in Fig. 8c. The bipartite graph in Fig. $7 \mathrm{~b}$ resumes the perfect ellipse matching.

Due to the amount of line segments in a scene, the line segment features are only used with the PTZ camera at the checking position, Fig. 8. The PTZ camera is directed towards the aircraft surface so the segments 


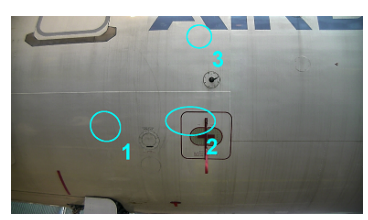

(a) Model features projected to the sensed image.

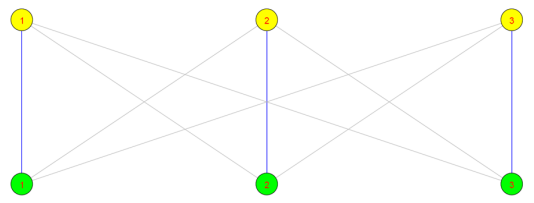

(b) Bipartite graph with eliminated edges (grey) and matches (blue).

Figure 7: Inspection mode.

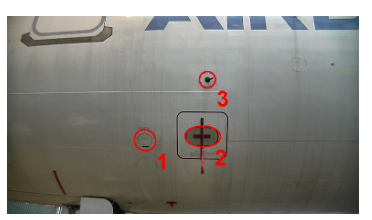

(c) Ellipse detection in the sensed image (EDCircles with the proposed postprocessing step).

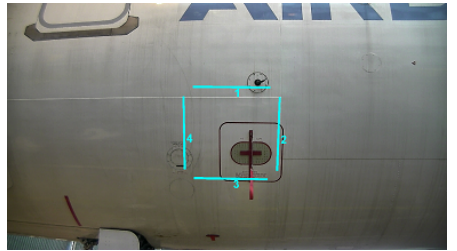

(a) Model features projected to the sensed image.

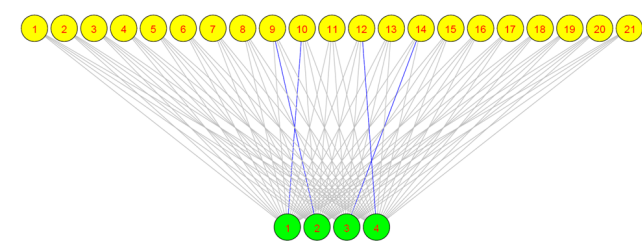

(b) Bipartite graph with eliminated edges (grey) and matches (blue)

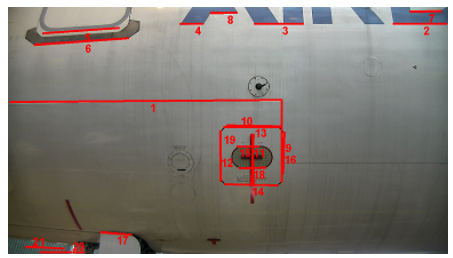

(c) Segment detection in the sensed image.

Figure 8: Inspection mode.

set is decreased. The line segments are used for the static port because sometimes the detection of the corresponding ellipse fails. The bipartite graph resumes the perfect segment matching, see Fig. $8 \mathrm{~b}$.

\section{INSPECTION APPROACHES}

In this section, the inspection approaches and numerical results will be provided for the air inlet vent and the static port.

Detection phase is evaluated by counting images on which the approach was able to isolate ROI successfully enough that the zooming is possible. Inspection approaches are evaluated by calculating false positive rate (FPR) and false negative rate (FNR). Input image in terms of the tested approach is classified as false positive (FP) in the case when the defect is not present and the approach reported a defect. It is classified as false negative $(\mathrm{FN})$ in the case when there is a defect and the method reports no defect.

False positive rate: ratio between number of false positive images and total number of images with no defect.

$$
F P R=\frac{F P}{F P+T N}
$$

where TN is the number of images with no defect on which the method reported no defect. FPR is the measure of false alarms.

False negative rate: ratio between number of false negative images and total number of images with de- fect.

$$
F N R=\frac{F N}{F N+T P}
$$

where TP is the number of images with defect on which the method reported a defect. FNR is the measure of missed defects. This measure is considered critical in our application.

\subsection{Air Inlet Vent}

The objective is to verify that the vent is closed (Fig. 9a). Challenging case is when the inlet is partially open (Fig. 9c). For a detailed inspection, a small sliding window is moved along the border of ROI (Fig. 9) and uniformity of the sliding window is estimated by the binarization.

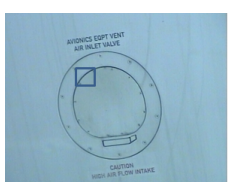

(a) Closed vent (negative).

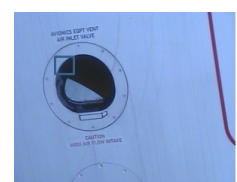

(b) Open vent (positive).

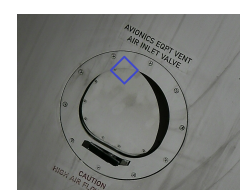

(c) Partially open vent (positive).

Figure 9: Air inlet vent - sliding window inspection.

In the case of open vent, there are two different regions within sliding window, while in the case of closed vent, the sliding window is uniform.

Matching algorithm was evaluated on a dataset of 23 low zoom images. It detected the vent in $100 \%$ of the 
cases. Inspection was evaluated on 32 high zoom (ex. Fig. 9) images (13 negative and 19 positive cases) of two different kinds of vent. On this dataset, the sliding window method performed with both FNR and FPR equal to $0 \%$.

\subsection{Static Ports}

The aim is to verify that the protecting cover is removed from the static ports (Fig. 10).

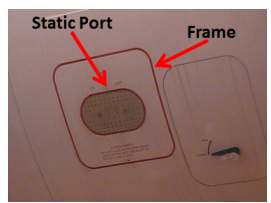

(a) Uncovered (negative).

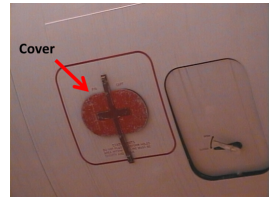

(b) Covered (positive).
Figure 10: F/O and CAPT static ports. Wrong situation is when protecting cover is still present (Fig. 10b).

Since there are red regions on the image, original RGB image is converted to CIELAB color space. A* channel of this color space is suitable for segmenting red regions. Further, from the input edge set, only edges corresponding to red regions are kept. The improvement can be seen in Fig. 11.

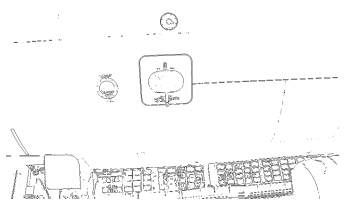

(a) All the edges of the input image.

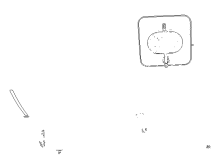

(b) Edges corresponding to red regions.
Figure 11: Significant reduction of edge set by rejecting edges of non-red regions.

On the zoomed image, segmentation based on region growing technique is employed on the static ports region, by setting the seed pixel in the mean position of the previously segmented rectangular ROI. Convexity of the detected static port region is used for making a decision. The region is convex in uncovered case (Fig. 12a) and concave in covered case (Fig. 12c). The convexity is estimated by the ratio of the segmented region area and the area of its convex hull.

The detection approach followed by the matching step was tested on a dataset of 75 images of two different kinds of static ports and it was successful in the $97 \%$ of the cases. The inspection method was evaluated on a dataset of 96 images (37 negative and 59 positive cases) with different cover shapes. The approach

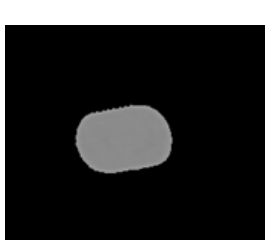

(a) Uncovered case: static ports region.

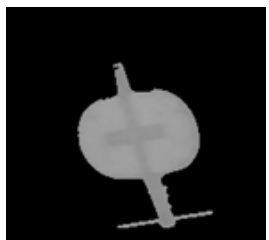

(c) Covered case: static ports region.

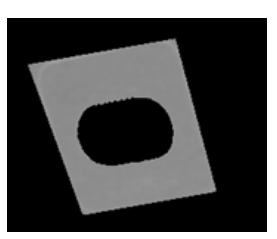

(b) Uncovered case: frame without static ports region.

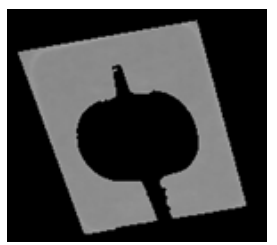

(d) Covered case: frame without static ports region.
Figure 12: Static ports - uncovered: both regions are not red; covered: region $12 \mathrm{c}$ is red, region $12 \mathrm{~d}$ is not red.

has shown accuracy with both FNR and FPR equal to $0 \%$. Detection and inspection results for both items are summarized in the Table 2.

Table 2: Evaluation results.

\begin{tabular}{|c|l|l|}
\hline \multicolumn{1}{|c|}{ Item: } & Air inlet vent & Static port \\
\hline Detection dataset & 23 & 75 \\
\hline Detection accuracy & $100 \%$ & $97 \%$ \\
\hline Inspection dataset & $\underline{32}$ negative & $\frac{96}{37}$ negative \\
& 19 positive & 59 positive \\
\hline Inspection FPR & $0 \%$ & $0 \%$ \\
\hline Inspection FNR & $0 \%$ & $0 \%$ \\
\hline
\end{tabular}

\section{CONCLUSION}

Similarity measure is used to match projected geometric primitives from CAD model with detected ones in real camera images. Existing similarity measure for segments is adapted to ellipses. This matching step facilitates the detection and data association processes for navigation and inspection tasks. In navigation mode, features are provided to the visual servoing and tracking algorithms. When robot is in checking area, location of the desired item is forwarded to the inspection algorithm. Results prove that even with an inaccurate robot location estimation, good matching results are still obtained so robot location can be corrected. It is important because the robot can switch 
from visual servoing to obstacle avoidance where the robot pose could be updated only with odometry. Minor problem has been noticed in some windows matching. The inclusion of distance information between features is in development. When the robot is really near the aircraft, ellipse extraction algorithm are not precise on the detection of the static port due to the fact that the shape is a superellipse. Superellipse feature extraction is under consideration.

Inspection approaches were presented for air inlet vent and static ports. Image processing approaches were evaluated on real airplane exterior images. On our dataset, inspection approaches could reach $0 \%$ FPR and FNR. For the moment, the approaches are adapted to the items and are based on heuristics. Comparison with binary classification methods is planned once the training data is sufficient.

Testing dataset is obtained in hangar with constant illumination. Some artificial illumination is added to make the dataset more representative. Next acquisition on tarmac with more various conditions (other airplanes present, weather, etc.) is planned.

\section{ACKNOWLEDGMENT}

This work is part of the AIR-COBOT project (http://aircobot.akka.eu) approved by the Aerospace Valley world competitiveness cluster. The authors would like to thank the French Government for the financial support via the Single Inter-Ministry Fund (FUI). The partners of the AIR-COBOT project (AKKA TECHNOLOGIES, Airbus Group, ARMINES, 2MoRO Solutions, M3 SYSTEMS and STERELA) are also acknowledged for their support.

\section{REFERENCES}

Akinlar, C. and Topal, C. (2011). EDLines: A real-time line segment detector with a false detection control. Pattern Recognition Letters, 32(13):1633 - 1642.

Akinlar, C. and Topal, C. (2013). EDCircles: A real-time circle detector with a false detection control. Pattern Recognition, 46(3):725 - 740.

Bay, H., Ferrari, V., and Gool, L. V. (2005). Wide-baseline stereo matching with line segments. Proceedings of Computer Vision and Pattern Recognition, 1:329-336.

Chaumette, F. and Hutchinson, S. (2006). Visual servo control, part i: Basic approaches. IEEE Robotics and $\mathrm{Au}$ tomation Magazine, 13(4):82-90.

Fishkel, F., Fischer, A., and Ar, S. (2006). Verification of engineering models based on bipartite graph matching for inspection applications. Springer-Verlag Berlin Heidelberg. LNCS 4077, pages 485-499.
Futterlieb, M., Cadenat, V., and Sentenac, T. (2014). A navigational framework combining visual servoing and spiral obstacle avoidance techniques. In Proc. of the 11th International Conference on Informatics in Control, Automation and Robotics, Volume 2, Vienna, Austria, 1 - 3 September, 2014, pages 57-64.

Hutter, M. and Brewer, N. (2009). Matching 2-d ellipses to 3-d circles with application to vehicle pose identification. Proc. of International Conference Image and Vision Computing, pages 153 - 158.

Jovančević, I., Larnier, S., Orteu, J.-J., and Sentenac, T. (2015). Automated exterior inspection of an aircraft with a pan-tilt-zoom camera mounted on a mobile robot. Journal of Electronic Imaging, 24(6):061110.

Jr., R. M. C., Bengoetxea, E., Bloch, I., and Larrañaga, P. (2005). Inexact graph matching for model-based recognition: Evaluation and comparison of optimization algorithms. Elsevier, Pattern Recognition, 38:2099-2113.

Malamas, E. N., Petrakis, E. G., Zervakis, M., Petit, L., and Legat, J.-D. (2003). A survey on industrial vision systems, applications and tools. Image and Vision Computing, 21(2): 171 - 188 .

Marchand, E. and Chaumette, F. (2005). Feature tracking for visual servoing purposes. Robotics and $\mathrm{Au}$ tonomous Systems, 52(1):53-70.

Matas, J., Galambos, C., and Kittler, J. (2000). Robust detection of lines using the progressive probabilistic hough transform. Computer Vision and Image Understanding, 78(1):119-137.

McIntosh, J. H. and Mutch, K. M. (1988). Matching straight lines. Computer Vision, Graphics and Image Processing, 43:386-408.

Mumtaz, R., Mumtaz, M., Mansoor, A. B., and Masood, H. (2012). Computer aided visual inspection of aircraft surfaces. International Journal of Image Processing (IJIP), 6(1):38-53.

Panda, B. and Pradhan, D. (2013). Minimum paireddominating set in chordal bipartite graphs and perfect elimination bipartite graphs. Journal of Combinatorial Optimization, 26:770 - 785 .

Prasad, D. K., Leung, M. K., and Quek, C. (2013). Ellifit: An unconstrained, non-iterative, least squares based geometric ellipse fitting method. Pattern Recognition, 46(5): 1449 - 1465.

Siegel, M., Gunatilake, P., and Podnar, G. (1998). Robotic assistants for aircraft inspectors. Instrumentation Measurement Magazine, IEEE, 1(1):16-30.

Viana, I., Bugarin, F., Cornille, N., and Orteu, J.-J. (2015). Cad-guided inspection of aeronautical mechanical parts using monocular vision.

von Gioi, R. G., Jakubowicz, J., Morel, J.-M., and Randall, G. (2010). LSD: A fast line segment detector with a false detection control. IEEE Transactions on Pattern Analysis and Machine Intelligence, 32(4):722-732. 